Fecha de recepción: 29 de julio de 2020

Sugerencia de citación: GarcíaSchilardi, M. E. (2021). Tamaño óptimo de las empresas de transporte colectivo que operan en el Área Metropolitana de Mendoza (Argentina), 2005-2012. tiempo\&economía, 8(1), 71-94. https://doi.org/10.21789/24222704.1631
Vol. 8 N. ${ }^{\circ} 1$

Enero - Junio del 2021

pp. $71-94$

DOI:

https://doi.org/10.21789/24222704.1631

\title{
Tamaño óptimo de las empresas de transporte colectivo que operan en el Área Metropolitana de Mendoza (Argentina), 2005-2012
}

\section{Optimum Size of the Collective Transportation Companies Operating in Mendoza Metropolitan Area (Argentina), 2005-2012}

\author{
María Emilia García Schilardi \\ Consejo Nacional de Investigaciones Científicas y Técnicas (CONICET), \\ Universidad Nacional de Cuyo, Argentina \\ https://orcid.org/0000-0003-4673-8142 \\ maria-emilia-garcia@hotmail.com
}

\section{RESUMEN}

Este trabajo avanza en el conocimiento de la estructura productiva del servicio de transporte colectivo en el Área Metropolitana de Mendoza (Argentina) durante la etapa previa a un cambio estructural del sistema de movilidad. Para ello, se estudia la existencia de economías de escala a través de la estimación de una función de costos de tipo translog. Se usan datos de panel para las empresas operadoras del servicio mediante buses, en el 
período 2005-2012. El resultado de este estudio evidencia un mercado que opera en el tramo de producción con deseconomías de escala, donde los costos promedios son crecientes, por lo que se recomienda que el mismo opere a través de un mayor número de empresas de menor tamaño.

Palabras clave: concentración, costos, economías de escala, transporte colectivo, historia económica.

Código JEL: R41.

\section{ABSTRACT}

This work makes contributions regarding the knowledge of the productive structure of the collective transport service in the Mendoza Metropolitan Area (Argentina) during the stage prior to a structural change in its mobility system. The existence of economies of scale is studied through the estimation of a translog cost function, using panel data for bus operating companies during the period 2005-2012. The results of this research show a market that operates in the production section with diseconomies of scale, where the average costs are increasing. Therefore, it is recommended for this system to operate through a larger number of small companies.

Keywords: Concentration, costs, scale economies, collective transport, economic history.

JEL Codes: R41. 


\section{INTRODUCCIÓN}

La movilidad urbana juega un papel fundamental en el desarrollo de las ciudades y sociedades modernas. Esta se ha convertido en una necesidad básica, puesto que posibilita la realización de actividades laborales, educativas, recreativas y culturales, entre otras. Bajo estas consideraciones, se torna fundamental conocer la estructura de mercado del servicio de transporte colectivo, componente clave de la movilidad. El comportamiento de este mercado puede evaluarse a través de la presencia o ausencia de economías de escala que resultan relevantes debido a que influyen en el nivel de las tarifas, en los costos, la producción y la organización de la industria (Berechman \& Giuliano, 1985). Este abordaje da la pauta de si conviene que exista mayor concentración de prestatarios o, por el contrario, una atomización de estos.

El objetivo de este trabajo es detectar la existencia de economías de escala en el servicio de transporte colectivo en el Área Metropolitana de Mendoza (Argentina) durante la etapa previa a un cambio estructural del sistema de movilidad. De esta manera, se puede evaluar si dicho cambio fue favorable o no en términos productivos. Esto facilita un conocimiento más profundo de la estructura productiva de dicho servicio, proporcionando herramientas para conducir la política pública relacionada. Además, esta investigación resulta de gran interés práctico, dado que en el territorio estudiado no existen precedentes con respecto al abordaje de estos temas. Así mismo, si bien la teoría económica es clara respecto de las condiciones necesarias para la existencia de economías de escala, en el mercado de transporte colectivo, en particular, no es posible definir un comportamiento general o la presencia o ausencia de dichas condiciones. Es decir, debe evaluarse cada caso y sus características propias. El presente trabajo muestra que el servicio analizado presenta condiciones y un comportamiento divergente respecto del que opera en otros territorios, dando cuenta de la imposibilidad de generalizar la existencia de dichas economías de escala.

Se elige el período 2009-2012 dado que este abarca desde el comienzo de la última licitación vigente (año 2005) hasta el año de la incorporación de modificaciones en el sistema de transporte de manera más estructural, principalmente con la inclusión del tranvía (2012) como modo adicional de transporte colectivo. Las licitaciones en el área de estudio se realizan cada 10 años. La que se otorgó en 2005 debería haber finalizado en 2015, pero la misma se 
prorrogó por 5 años más, hasta 2020. Así, en el transcurso de estos 15 años se realizaron modificaciones relevantes en el sistema de transporte colectivo; en 2012 se pone en marcha el servicio de metro tranvías y en 2019 se realiza una modificación integral de los recorridos y frecuencias del sistema mediante buses. Este trabajo aporta conocimiento sobre la estructura productiva del servicio de transporte colectivo justo en el período anterior a cualquier modificación, lo cual permite sentar bases para evaluar los cambios generados y elaborar una política de movilidad más eficiente a partir de la nueva licitación.

El trabajo se plantea desde la óptica de la teoría económica aplicada al transporte. Por ello, no solo se emplean técnicas metodológicas correspondientes a esta perspectiva, sino que el análisis de los resultados también se realiza a partir de los argumentos y conceptos de esta teoría. La búsqueda tiene que ver con las causas y los factores que generan esta situación, pudiendo ser internas o externas a cada firma. Así mismo, se fundamenta la importancia del conocimiento de este mercado, particularmente a partir del enfoque de la movilidad urbana sostenible, el cual focaliza su atención en el desarrollo óptimo de este servicio, que se constituye como uno de los principales elementos de desarrollo urbano.

Para el logro del objetivo, se emplea una técnica estadística que permite estimar la función de costos de las firmas a partir de una función de tipo translog y el uso datos de panel para las empresas operadoras del servicio mediante ómnibus en el período 2005-2012. De este modo, se busca mejorar la posición del Estado en cuanto a planificar adecuadamente y regular el servicio a fin de beneficiar a la mayor parte de la población.

El resultado de este estudio evidencia un mercado de transporte colectivo que opera en el tramo de producción con deseconomías de escala, donde los costos promedios son crecientes. Esto implica la necesidad de una oferta del servicio más atomizada, es decir, de mayor cantidad de empresas prestatarias de menor tamaño.

\section{REVISIÓN DE LA LITERATURA}

La presencia o ausencia de economías de escala en el mercado de transporte colectivo impacta directamente los mecanismos de regulación de la tarifa y de asignación y distribución de subsidios. De igual manera, esto influye en la estructura de la industria y en la asignación de costos (Troncoso, 2017). Tales factores muestran la fuerte incompatibilidad en las políticas públicas vinculadas a estos aspectos. 
Investigaciones empíricas en diversos países y distintos períodos de tiempo han arrojado resultados diferentes (economías de escala crecientes, constantes y decrecientes), como se muestra en la tabla 1. Según se observa en la literatura, no se puede establecer una conclusión general respecto a la presencia o ausencia de economías de escala en el mercado de transporte colectivo (Berechman \& Giuliano, 1985; Hensher, 1987; Lai et al., 2012). En estos términos, no es acertada, a priori, la afirmación de que las empresas de mayor tamaño sean más eficientes en la prestación del servicio que aquellas más pequeñas, puesto que este resultado depende de diversas variables con particularidades diferentes en el mercado de transporte colectivo de cada territorio. Según plantea Miller (1970), solo podrían compararse los niveles de producción en el de caso que todas las ciudades tuvieran la misma distribución de la población, la misma geografía y la misma actividad económica. Así mismo, los resultados dependen del tipo de base de datos disponible y de la metodología empleada. Por ejemplo, en los casos de estimaciones de funciones translog se observa que las elasticidades de costos obtenidas presentan gran variabilidad debido a las diferencias en las bases de datos que incluyen empresas operando en territorios diferentes. Evidencia de ello son los estudios antecedentes de Koshal (1970), Lee y Steedman (1970), Williams y Dalal (1981), Viton (1981), Tauchen et al. (1983), de Borger (1984), Matas y Raymond (1998) y Filippini y Prioni (2003). Otro aspecto que influye en la existencia de economías de escala es el marco regulatorio, ya que este puede afectar las funciones de producción de las prestatarias, dificultando su eficiencia (de Grange et al., 2018).

Por último, se detecta que entre los antecedentes no abundan los casos de territorios latinoamericanos, evidenciando un vacío en el análisis de estos mercados. El presente trabajo, por ende, busca aportar algo de luz al conocimiento sobre el asunto.

\section{MATERIALES Y MÉTODOS}

\section{Universo de análisis}

El servicio analizado opera en el Área Metropolitana de Mendoza, ubicada en la provincia de Mendoza, Argentina. Este territorio urbano se posiciona en el cuarto lugar de importancia en la jerarquización del sistema urbano argentino, de acuerdo con su población, dotación de equipamientos y funcionalidad. En la actualidad, en esta área, el sistema de transporte colectivo opera mediante dos modalidades: tranvía urbano y buses; siendo objeto de análisis solamente el servicio prestado mediante buses. 


\begin{tabular}{|c|c|c|}
\hline Autores & País de la muestra & Tipo de economías de escala \\
\hline Lee y Steedman (1970) & Gran Bretaña & Constantes \\
\hline Koshal (1970) & EE. UU. & Constantes \\
\hline Caves y Christensen (1988) & EE. UU. & Constantes \\
\hline de Rus y Nombela (1997) & España & Constantes \\
\hline Dalen y Gomez-Lobo (2003) & Dinamarca & Constantes \\
\hline Wabe y Coles (1975) & Gran Bretaña & Decrecientes \\
\hline Koenker (1977) & EE. UU. & Decrecientes \\
\hline McGillivray et al. (1980) & EE. UU. & Decrecientes \\
\hline Williams y Dalal (1981) & EE. UU. & Decrecientes \\
\hline Pucher et al. (1983) & EE. UU. & Decrecientes \\
\hline Morlok (1984) & EE. UU. & Decrecientes \\
\hline Obeng (1985) & EE. UU. & Decrecientes \\
\hline Perry y Babitsky (1986) & EE. UU. & Decrecientes \\
\hline Cervero (1988) & EE. UU. & Decrecientes \\
\hline Shughart y Kimenyi (1991) & EE. UU. & Decrecientes \\
\hline Savage (1993) & Gran Bretaña & Decrecientes \\
\hline Xu et al. (1994) & EE. UU. & Decrecientes \\
\hline Jørgensen et al. (1997) & Dinamarca & Decrecientes \\
\hline Matas y Raymond (1998) & España & Decrecientes \\
\hline McCullough et al. (1998) & EE. UU. & Decrecientes \\
\hline Sclar (2000) & EE. UU. & Decrecientes \\
\hline Fernández et al. (2005) & Chile & Decrecientes \\
\hline Iseki (2008) & EE. UU. & Decrecientes \\
\hline Ayadi y Hammami (2015) & Túnez & Decrecientes \\
\hline de Grange et al. (2018) & Chile & Decrecientes \\
\hline Williams (1979) & EE. UU. & Crecientes \\
\hline Viton (1981) & EE. UU. & Crecientes \\
\hline
\end{tabular}

Tabla 1.

Antecedentes sobre economías de escala en la literatura
Fuente: elaboración propia con base en de Grange et al. (2018), Cubukcu (2008), de Rus (2006) y Jara-Díaz (1982). 


\begin{tabular}{|c|c|c|}
\hline Williams y Hall (1981) & EE. UU. & Crecientes \\
\hline Berechman (1983) & Israel & Crecientes \\
\hline Hensher (1987) & Australia & Crecientes \\
\hline Sakano y Obeng (1995) & EE. UU. & Crecientes \\
\hline Karlaftis y McCarthy (2002) & EE. UU. & Crecientes \\
\hline Filippini y Prioni (2003) & Suiza & Crecientes \\
\hline Sha-Er et al. (2005) & Taiwán & Crecientes \\
\hline Cubukcu (2006) & Turquía & Crecientes \\
\hline Farsi et al. (2007) & Suiza & Crecientes \\
\hline Walter (2011) & Alemania & Crecientes \\
\hline Fiorio et al. (2013) & Europa & Crecientes \\
\hline Tauchen et al. (1983) & EE. UU. & Resultados mixtos \\
\hline De-Borger (1984) & Bélgica & Resultados mixtos \\
\hline Berechman y Giuliano (1985) & EE. UU. & Resultados mixtos \\
\hline Button y O'Donnell (1985) & Gran Bretaña & Resultados mixtos \\
\hline Berechman (1993) & $\begin{array}{l}\text { EE. UU., Australia, Nueva Zelanda, } \\
\text { Japón, Europa }\end{array}$ & Resultados mixtos \\
\hline Cowie y Asenova (1999) & Reino Unido & Resultados mixtos \\
\hline Singh (2002) & India & Resultados mixtos \\
\hline Filippini y Prioni (2003) & Suiza & Resultados mixtos \\
\hline Lai et al. (2012) & Hong Kong & Resultados mixtos \\
\hline Abrate et al. (2016) & Italia & Resultados mixtos \\
\hline Avenali et al. (2016) & Italia & Resultados mixtos \\
\hline
\end{tabular}

Los prestatarios del servicio mediante buses entran al mercado a través de una licitación. En 1965 se realiza la primera licitación del servicio, siendo esta renovada en 1979 por 10 años más. No obstante, en 1991 se realiza la tercera licitación del servicio con cambios estructurales en lo referido a recorridos y situación formal de las prestatarias. En 2005 se lleva adelante la cuarta licitación, la cual mantiene las características establecidas en la 
anterior concesión y está vigente hasta 2020. La duración estipulada para las adjudicaciones no ha sido respetada en ningún caso y la situación general del servicio se mantiene relativamente constante en el período que va de 1991 a 2012. En este último año se pone en marcha el servicio de tranvía urbano y luego, en 2019, se realiza una reordenación del sistema de movilidad. Se toma como espacio de tiempo estudiado el período que va desde el año 2005, comienzo de la cuarta licitación, hasta 2012, año de aparición de otro modo masivo de transporte.

A través del contrato de licitación con el Estado, los oferentes resultan regulados y controlados respecto de los recorridos, el modo de cálculo de la tarifa, las frecuencias de cada línea, las características técnicas del parque móvil y su plan de renovación, las emisiones contaminantes, los subsidios a la demanda, las exigencias legales y contables y la adhesión al sistema prepago del boleto, cuya recaudación está tercerizada y cuya distribución se da a través de los kilómetros recorridos por cada vehículo. Esto último implica que es el Estado el que asume todo el riesgo, limitándose las concesionarias a cumplir con las condiciones que este impone.

El servicio se adjudica por grupos de recorridos, pudiéndose presentar una misma empresa como candidata para la concesión en más de un grupo. En la actualidad existen 10 grupos de recorridos, cuyos servicios son prestados por siete empresas de transporte (tabla 2), siendo una sola empresa la adjudicataria de tres de estos grupos. En la licitación anterior existía mayor cantidad de recorridos y de adjudicatarios. Es decir, la oferta de mercado se ha concentrado a lo largo del tiempo.

Las firmas adjudicatarias reciben subsidios provinciales y nacionales, que se constituyen como subsidios a la oferta sin condicionamientos; es decir, para su otorgamiento no se exigen requisitos previos a cumplir. Los subsidios nacionales buscan compensar los incrementos de costos sufridos por las empresas concesionarias, mientras que los subsidios provinciales suplen el déficit existente entre la recaudación del sistema y los subsidios nacionales. Así mismo, las prestatarias se ven beneficiadas por un cupo de gasoil a precio diferencial calculado a partir del gasto total en combustible que cada empresa declara por los kilómetros que recorre.

En este contexto, las firmas prestatarias parecen no tener incentivos para la eficiencia, no asumen riesgos y operan en un mercado cada vez más concentrado, por lo que cada una de ellas tiene una cuota mayor del mismo y recibe subsidios que no requieren ninguna condición previa o, en otras 
palabras, no generan incentivos para un funcionamiento óptimo del servicio. Frente a esta situación, los requerimientos establecidos en los pliegos licitatorios pueden resultar un modo de guiar a la estructura de mercado hacia un punto más cercano al equilibrio socialmente óptimo. Por ende, identificar si en este mercado es mejor una mayor atomización o concentración de la oferta es clave para diseñar las próximas condiciones de entrada al mismo.

Tabla 2. Empresas prestadoras del

\begin{tabular}{|c|c|c|}
\hline & Licitación 1991-2005 & Licitación 2005-2020 \\
\hline $\begin{array}{l}\text { Grupo de } \\
\text { recorridos }\end{array}$ & Empresas operadoras & Empresas operadoras \\
\hline 1 & El Trapiche S.R.L. & El Trapiche S.R.L. \\
\hline 2 & Transportes Antártida S. A. & Empresa Maipú S.R.L. \\
\hline 3 & Autotransportes Presidente Alvear & $\begin{array}{c}\text { UTE: Autotransportes Presidente Alvear, } \\
\text { Autotransportes Los Andes, Autotrans- } \\
\text { portes Gral. Roca }\end{array}$ \\
\hline 4 & El Trapiche S.R.L. & $\begin{array}{l}\text { UTE: El Trapiche S.R.L., Autotransportes } \\
\text { Los Andes }\end{array}$ \\
\hline 5 & Autotransportes Gral. Roca & Autotransportes Gral. Roca \\
\hline 6 & El Plumerillo S.A. & El Plumerillo S.A. \\
\hline 7 & $\begin{array}{c}\text { Autotransportes Bartolomé Matienzo } \\
\text { S. A. }\end{array}$ & El Cacique S.A. \\
\hline 8 & Autotransportes Los Andes S. A. & El Cacique S.A. \\
\hline 9 & Transportes Colectivos del Oeste S.A. & El Cacique S.A. \\
\hline 10 & El Cacique S.A. & Empresa Maipú S.R.L. \\
\hline 11 & Transporte Coronel Díaz S.A. & - \\
\hline 12 & $\begin{array}{c}\text { Autotransportes Bartolomé Matienzo } \\
\text { S. A. }\end{array}$ & - \\
\hline 13 & El Cacique S.A. & - \\
\hline 14 & Empresa Maipú S.R.L. & - \\
\hline 15 & Empresa Maipú S.R.L. & - \\
\hline $\begin{array}{c}\text { Total } \\
\text { prestatarias }\end{array}$ & 11 & 7 \\
\hline
\end{tabular}

\section{Metodología empleada}

Debido a la variabilidad en los resultados sobre la presencia o ausencia de economías de escala en este tipo de servicio en los diversos territorios y a las características del mercado estudiado, se detallan a continuación las particularidades tanto de los datos como de la metodología empleados. 


\section{Datos empleados}

Los datos con los que se elaboró este trabajo provienen de diversas fuentes. Los conceptos más relevantes para la estructura de costos se determinaron según postula la teoría microeconómica. Por otro lado, los montos se obtuvieron de la información proporcionada por la Secretaría de Transporte de la Provincia de Mendoza (2012), la Asociación Unida Transporte Automotor Mendoza y la Secretaría de Energía de la Nación (2012) (particularmente para los precios del combustible).

La base de datos de panel incluye 80 observaciones, pertenecientes al período 2005-2012, y el corte transversal corresponde a las empresas de transporte de ómnibus que operan en el área metropolitana de Mendoza. Debido a que en el período analizado ha existido un fuerte proceso inflacionario, se han ajustado los valores por dicha inflación con base en el año 2005. Se ha considerado un promedio del índice elaborado por consultoras privadas y no aquel de estadísticas oficiales, en tanto el primero se considera más adecuado a la realidad analizada. El output está expresado en kilómetros recorridos por año por cada empresa. La tabla 3 presenta las estadísticas descriptivas de cada variable considerada. El costo total anual en promedio, con base de precios en el año 2005, es de ARS 21.822.767.

\begin{tabular}{|c|c|c|c|c|c|}
\hline Variable & $\begin{array}{c}\text { N. }{ }^{\circ} \text { de observa- } \\
\text { ciones }\end{array}$ & Media & $\begin{array}{c}\text { Desviación } \\
\text { estándar }\end{array}$ & Mínimo & Máximo \\
\hline $\begin{array}{c}\text { Precio anual por } \\
\text { bus }\end{array}$ & 80 & 89.722 & 16.427 & 54.040 & 132.941 \\
\hline $\begin{array}{c}\text { Precio gasoil por } \\
\text { litro }\end{array}$ & 80 & 2,49 & 1,16 & 1,24 & 4,79 \\
\hline $\begin{array}{c}\text { Precio por hora } \\
\text { del personal de } \\
\text { conducción }\end{array}$ & 80 & 8,97 & 2,31 & 4,18 & 11,52 \\
\hline $\begin{array}{c}\text { Kilómetros reco- } \\
\text { rridos por año }\end{array}$ & 80 & 7.077 .743 & 1.854 .918 & 2.769 .676 & 9.500 .158 \\
\hline Costo total & - & 21.822 .767 & 7.168 .590 & 7.218 .270 & 36.580 .832 \\
\hline
\end{tabular}

\section{Modelo considerado}

Entre los elementos que forman parte de la estructura productiva de un mercado se encuentra el tamaño que alcanzan las firmas que operan en el mismo. Este depende de varios factores internos y externos a la misma, que originan lo que en economía se llama economías o deseconomías de escala. El primer concepto hace referencia a la capacidad de una empresa de aumentar su producción reduciendo el costo por cada unidad producida.
Tabla 3.

Estadísticas descriptivas de las variables analizadas (cifras en pesos argentinos, ARS)

Fuente: elaboración propia en el software estadístico STATA/IC. 
La existencia de esta situación implica que es más eficiente una empresa o pocas empresas de mayor tamaño en vez de varias de menor tamaño. El segundo concepto hace referencia a la inexistencia de esta capacidad, por lo que resulta más eficiente que existan varias empresas de menor tamaño.

Para evaluar la existencia o no de economías de escala, se analiza la función de producción o la función de costos de las firmas. Según ha demostrado Shepard (1970), las funciones de costos y de producción son duales bajo ciertas condiciones de regularidad. No obstante, es más habitual la estimación empírica de las funciones de costos, ya que estas resumen toda la información económicamente importante y a la vez tienen ventajas en el proceso de estimación. Esto último, relacionado principalmente a que estas funciones proveen un modo más directo de estimar las economías de escala (Christensen \& Greene, 1976).

Como elementos comunes del mercado en el que operan las compañías, se observa lo siguiente:

- El nivel de producción es exógeno, lo cual hace más atractiva la estimación de las funciones de costos (Christensen \& Greene, 1976). Esto se debe a que los operadores de transporte toman como dada la demanda de servicio que se define en el proceso regulatorio.

- Las rutas y tarifas están también determinadas por la autoridad estatal competente.

- Ninguna empresa concesionaria tiene poder para afectar los precios de los factores productivos. Todas son tomadoras de precios en el mercado de inputs.

De esta manera, cada empresa minimiza sus costos y las variables de decisión son las cantidades de insumos necesarias para suplir la demanda exógena (Cubukcu, 2008).

La función de costos de los productores de servicios de transporte (ecuación 1) viene dada por la siguiente expresión:

$$
C=F(Q, P)
$$

Donde $C$ es el costo total, $Q$ el nivel de producción del servicio y $P$ los precios de los factores productivos.

Esta es una función general. Para conocer las especificaciones funcionales del caso en estudio, se realiza una estimación a partir de datos, para lo cual se pueden emplear diversos métodos. 
Para estimar esta función, en este trabajo se emplea la especificación funcional translog, que es una aproximación de Taylor de segundo orden de la función desconocida, y se estima a través de mínimos cuadrados generalizados. Se asume que cada empresa de transporte produce sólo un tipo de servicio medido en kilómetros totales recorridos en un año y utiliza tres insumos: personal de conducción (trabajo), autobuses (capital) y gasoil (combustible).

La especificación elegida para la función de costos (ecuación 2) es:

$\operatorname{LnCT}=\alpha+\beta_{Q} \operatorname{Ln} Q+\beta_{L} \operatorname{Ln} P_{L}+\beta_{k} \operatorname{Ln} P_{k}+\beta_{G} \operatorname{Ln} P_{G}+(1 / 2) \beta_{Q Q}[(\operatorname{Ln} Q)]^{2}+(1 / 2) \beta_{L L}\left[\left(\operatorname{Ln} P_{L}\right)\right]^{2}+$ $(1 / 2) \beta_{K K}\left[\left(\operatorname{Ln} P_{K}\right)\right]^{2}+(1 / 2) \beta_{G G}\left[\left(\operatorname{Ln} P_{G}\right)\right]^{2}+\beta_{L K}\left(\operatorname{Ln} P_{L}\right)\left(\operatorname{Ln} P_{K}\right)+\beta_{L G}\left(\operatorname{Ln} P_{L}\right)\left(\operatorname{Ln} P_{G}\right)+\beta_{K G}$ $\left(\operatorname{Ln} P_{K}\right)$

$\left(\operatorname{Ln} P_{G}\right)+\beta_{Q L}(\operatorname{Ln} Q)\left(\operatorname{Ln} P_{L}\right)+\beta_{Q K}(\operatorname{Ln} Q)\left(\operatorname{Ln} P_{K}\right)+\beta_{Q G}(\operatorname{Ln} Q)\left(\operatorname{Ln} P_{G}\right)$

Donde $C T$ es el costo total anual, $Q$ es el nivel de producción de servicio medido en kilómetros totales recorridos por año, $P_{K}$ es el precio unitario del capital medido como el costo total anual de un autobús, $P_{L}$ el precio unitario del trabajo medido como el costo por hora en personal de conducción y $P_{G}$ el precio unitario de combustible medido como el costo por litro de gasoil.

De la aplicación del lema de Shepard a la función de costos translog se obtienen las participaciones de los costos (cost shares) de cada factor (ecuación 3).

$S_{L}=\delta \operatorname{LnCT} /\left(\delta \operatorname{Ln} P_{L}=\right) \beta_{L}+\beta_{L L} \operatorname{Ln} P_{L}+\beta_{K L} \operatorname{Ln} P_{K}+\beta_{L G} \operatorname{Ln} P_{G}+\beta_{Q L} \operatorname{Ln} Q$

$S_{K}=\delta \operatorname{LnCT} /\left(\delta \operatorname{Ln} P_{K}=\right) \beta_{K}+\beta_{K K} \operatorname{Ln} P_{K}+\beta_{K L} \operatorname{Ln} P_{L}+\beta_{K G} \operatorname{Ln} P_{G}+\beta_{Q K} \operatorname{Ln} Q$

$S_{G}=\delta \operatorname{LnCT} /\left(\delta \operatorname{Ln} P_{G}=\right) \beta_{G}+\beta_{G G} \operatorname{Ln} P_{G}+\beta_{K G} \operatorname{Ln} P_{K}+\beta_{L G} \operatorname{Ln} P_{L}+\beta_{Q G} \operatorname{LnQ}$

Es habitual que en los fenómenos económicos se determinen simultáneamente un conjunto de variables. En este caso, dichas variables son el costo y las demandas de insumos en un modelo de minimización de costos. Esta simultaneidad implica relaciones teóricas entre las ecuaciones que describen tales variables. En particular, la aplicación del Lema de Shepard para obtener las participaciones relativas de los factores indica que los parámetros que aparecen en estas ecuaciones sean los mismos que en la función de costos. Dado que las ecuaciones del sistema comparten los mismos parámetros (o un subconjunto de ellos), es frecuente que se estimen conjuntamente, basados en argumentos econométricos de mayor eficiencia en la estimación. 
Bajo esta consideración, el procedimiento óptimo es estimar la función de costos y las funciones de participación de los insumos de forma conjunta como un sistema de regresión multivariada. De esta forma, se adicionan grados de libertad sin agregar coeficientes a la regresión, lo que resulta en una estimación de los parámetros más eficiente, comparada con los obtenidos por mínimos cuadrados ordinarios (Christensen \& Greene, 1976).

Se imponen las restricciones paramétricas de igualdad de coeficientes y de homogeneidad de la función de costos. Dado que la suma de las ecuaciones de participación de los factores es igual a uno, se elimina una de las ecuaciones del sistema para que la matriz de covarianzas no sea singular. Se asume, así mismo, que: (i) los términos de error tienen una distribución conjunta normal (Christensen \& Greene, 1976); (ii) no existe correlación entre las firmas; y (iii) se permiten correlaciones no nulas de una firma particular (Zellner, 1962).

\section{RESULTADOS Y DISCUSIÓN}

\section{Estimación de la función de costos}

La tabla 4 presenta la estimación de tres modelos alternativos. Esto implica que a partir del mismo método de estimación se analizan tres especificaciones funcionales diferentes, tres modelos, y se evalúa cuál de ellas se ajusta mejor a los datos observados.

El modelo $A$ corresponde a una función de costos Cobb Douglas, que implica homoteticidad $\left(\beta_{\mathrm{QL}}=\beta_{\mathrm{QK}}=\beta_{\mathrm{QG}}=0\right.$ ), es decir, separabilidad entre el output y los factores. Este excluye términos de segundo orden para los precios de los insumos y de la producción ( $\beta_{\mathrm{QQ}}=\beta_{\mathrm{LL}}=\beta_{\mathrm{KK}}=\beta_{\mathrm{GG}}=0$ ). Por su parte, el modelo $B$ corresponde a una función de producción homotética. Impone restricciones de separabilidad entre insumos y producción, pero incluye términos de segundo orden para precio de factores y de producción. El modelo $C$ es el modelo completo, y no asume separabilidad entre insumos y output ni elasticidad de sustitución entre factores constante, este incluye términos de segundo orden para precios de inputs y producción.

Se detecta que la bondad de ajuste del modelo $B\left(R^{2}\right.$ ajustado) es menor a la de los modelos A y C. Por lo tanto, la decisión del modelo a elegir está entre estas dos últimas alternativas. Si bien bajo este criterio debiera elegirse el modelo $C$, se realiza el test de likelihood para evaluar la diferencia 


\begin{tabular}{|c|c|c|c|}
\hline \multirow{2}{*}{ Coeficientes } & \multicolumn{3}{|c|}{ Modelos } \\
\hline & A & B & C \\
\hline \multirow[t]{2}{*}{ a } & 1,7557 & 12,6847 & 13,8355 \\
\hline & 12,84 & 2,99 & 3,39 \\
\hline \multirow[t]{2}{*}{$\boldsymbol{\beta}_{\mathrm{Q}}$} & 0,9594 & $-0,4648$ & $-0,4716$ \\
\hline & 110,09 & $-0,85$ & $-0,90$ \\
\hline \multirow[t]{2}{*}{$\boldsymbol{\beta}_{\mathbf{L}}$} & 0,4684 & 0,5166 & 0,7764 \\
\hline & 26,92 & 27,10 & 13,21 \\
\hline \multirow[t]{2}{*}{$\boldsymbol{\beta}_{\mathrm{k}}$} & 0,2812 & 0,2691 & 0,6432 \\
\hline & 13,80 & 12,13 & 8,16 \\
\hline \multirow[t]{2}{*}{$\boldsymbol{\beta}_{\mathrm{G}}$} & 0,2504 & 0,2144 & $-0,4196$ \\
\hline & 17,24 & 13,47 & $-3,97$ \\
\hline \multirow[t]{2}{*}{$\beta_{Q 0}$} & & 0,0926 & 0,0842 \\
\hline & & 2,61 & 2,47 \\
\hline \multirow[t]{2}{*}{$\boldsymbol{\beta}_{\mathrm{LL}}$} & & $-0,1026$ & $-0,1069$ \\
\hline & & $-17,86$ & $-18,48$ \\
\hline \multirow[t]{2}{*}{$\boldsymbol{\beta}_{\mathrm{KK}}$} & & $-0,1283$ & $-0,1192$ \\
\hline & & $-23,05$ & $-22,64$ \\
\hline \multirow[t]{2}{*}{$\boldsymbol{\beta}_{\mathrm{GG}}$} & & 0,3427 & 0,3403 \\
\hline & & 9,26 & 9,61 \\
\hline \multirow[t]{2}{*}{$\beta_{\mathrm{LK}}$} & & 0,2461 & 0,2403 \\
\hline & & 60,22 & 59,00 \\
\hline \multirow[t]{2}{*}{$\boldsymbol{\beta}_{\mathrm{LG}}$} & & $-0,1337$ & $-0,1270$ \\
\hline & & $-34,85$ & $-33,54$ \\
\hline \multirow[t]{2}{*}{$\boldsymbol{\beta}_{\mathrm{KG}}$} & & $-0,1124$ & $-0,1133$ \\
\hline & & $-44,52$ & $-43,75$ \\
\hline \multirow[t]{2}{*}{$\boldsymbol{\beta}_{\mathrm{QL}}$} & & & $-0,0187$ \\
\hline & & & $-4,82$ \\
\hline \multirow[t]{2}{*}{$\boldsymbol{\beta}_{\mathrm{QK}}$} & & & $-0,0231$ \\
\hline & & & $-4,45$ \\
\hline \multirow[t]{2}{*}{$\boldsymbol{\beta}_{\mathrm{QG}}$} & & & 0,0418 \\
\hline & & & 6,17 \\
\hline $\mathrm{R}^{2}$ Ecuación de Costos & 0,9454 & 0,9391 & 0,9611 \\
\hline
\end{tabular}

Tabla 4.

Estimación de la función de costos (t estadístico debajo de cada coeficiente estimado)

entre modelos anidados y poder seleccionar así la estimación que mejor explica el comportamiento de la función de costos. Un modelo está anidado en otro si este se genera a partir de la imposición de restricciones sobre los parámetros del primero. Habitualmente, la restricción que se impone es que sean iguales a cero. Este es el caso en análisis. El test de likelihood compara los log likelihood de los dos modelos y evalúa si la diferencia es estadísticamente significativa. Si esto ocurre, entonces, el modelo con menores restricciones 
se adecúa mejor a los datos que el modelo más restrictivo. Dado que para el caso en estudio la diferencia no es estadísticamente significativa ( $p$, valor asociado considerablemente bajo, 9.476e-11), se concluye que el modelo C es el que mejor se adecúa al comportamiento de los datos de la muestra.

En otras palabras, de los tres modelos considerados se observa que $A$ y $C$ explican de mejor manera la conformación de los costos (esto lo indica el mayor valor del $R^{2}$ ajustado). El modelo $B$ es una forma funcional menos precisa en la explicación del comportamiento de los costos que los otros dos modelos. Entre los modelos $\mathrm{A}$ y $\mathrm{C}$ se analiza si el último se construye a partir del primero; es decir, si el modelo A es un caso particular del modelo C. Se realiza una prueba y se observa que son modelos separados, por lo que se elige el modelo $C$ como aquel que se ajusta mejor al modo en que se distribuyen los datos observados.

Al realizar estimaciones de los parámetros se pueden observar algunos problemas que resulta importante sean detectados y solucionados para que dicha estimación sea eficiente, insesgada y consistente. Por un lado, puede ocurrir que se omitan variables relevantes o se incluyan variables irrelevantes en el modelo. En el caso analizado se asume que este problema no ocurre, dado que la estimación está respaldada por abundante teoría económica que fundamenta las variables elegidas como explicativas del fenómeno económico. Por otro lado, se requiere la existencia de homocedasticidad. En el caso de estudio, dicha variable viene garantizada por la adecuada especificación del modelo y por la estimación de los coeficientes a través de mínimos cuadrados generalizados. Finalmente, se debe descartar el problema de multicolinealidad. Para el caso en estudio se obtienen todos los coeficientes de todos los pares de variables independientes para evaluar la colinealidad. Se detecta algún grado de correlación entre algunas de ellas. Sin embargo, dado que esta relación no es de multicolinealidad exacta y que los signos del modelo estimado son como los esperados y estadísticamente significativos, se asume que no es un problema relevante.

\section{Resultados}

Los signos de los coeficientes del modelo son como se esperaban y todos estadísticamente significativos en un nivel de $5 \%$, es decir, existe evidencia estadística de que la variable que acompañan tiene relevancia en la explicación de los costos. La forma funcional estimada (ecuación 4) es la siguiente: 
Ln $C T=13,8355+0,7764 \operatorname{Ln} P_{L}+0,6432 \operatorname{Ln} P_{K}-0,4196 \operatorname{Ln} P_{G}+0,0842(1 / 2) \beta_{Q Q}[(\operatorname{LnQ})$

]$^{2}-0,1069(1 / 2)\left[\left(\operatorname{Ln} P_{L}\right)\right]^{2}-0,1192(1 / 2)\left[\left(\operatorname{Ln} P_{K}\right)\right]^{2}+0,3403(1 / 2)\left[\left(\operatorname{Ln} P_{G}\right)\right]^{2}+0,2403\left(\operatorname{Ln} P_{L}\right)$

$\left(\operatorname{Ln} P_{K}\right)-0,1270\left(\operatorname{Ln} P_{L}\right)\left(\operatorname{Ln} P_{G}\right)-0,1133\left(\operatorname{Ln} P_{K}\right)\left(\operatorname{Ln} P_{G}\right)-0,0187(\operatorname{Ln} Q)\left(\operatorname{Ln} P_{L}\right)-0,0231(\operatorname{Ln} Q)$

$\left(\operatorname{Ln} P_{K}\right)+0,0418(\operatorname{Ln} Q)\left(\operatorname{Ln} P_{G}\right)$

Y las funciones de participación de los factores (ecuación 5) son:

$S_{L}=\delta \operatorname{LnCT} /\left(\delta \operatorname{Ln} P_{L}=\right) 0,7764-0,1069 \operatorname{Ln} P_{L}+0,2403 \operatorname{Ln} P_{K}-0,127 \operatorname{Ln} P_{G}-0,0187 \operatorname{Ln} Q$

$S_{K}=\delta \operatorname{LnCT} /\left(\delta \operatorname{Ln} P_{K}=\right) 0,6432-0,1192 \operatorname{Ln} P_{K}+0,2403 \operatorname{Ln} P_{L}-0,1133 \operatorname{Ln} P_{G}-0,231 \operatorname{Ln} Q$

$S_{G}=\delta \operatorname{LnCT} /\left(\delta \operatorname{Ln} P_{G}=\right)-0,4196+0,3403 \operatorname{Ln} P_{G}-0,1133 \operatorname{Ln} P_{K}-0,127 \operatorname{Ln} P_{L}+0,0418 \operatorname{Ln} Q$

(Ec. 5)

El concepto que se usa para evaluar la existencia de economías de escala es la elasticidad costo con respecto a la producción, que indica la variación porcentual que se da en los costos ante un cambio de $1 \%$ en la producción. Cuando este valor es mayor a 1 significa que existen deseconomías de escala; es decir, el cambio en costos es más que proporcional al cambio en la producción. En este caso, la elasticidad del costo con respecto a la producción varía entre 1,2355 y 1,3747, con una media de 1,3241. (ecuación 6):

${ }^{\varepsilon} C, Q=C M g / C M e=(\delta C / \delta Q) *(Q / C)=\delta \operatorname{LnCT} / \delta \operatorname{Ln} Q=0,0842(L Q)-$ $0,0187\left(\operatorname{Ln} P_{L}\right)-0,0231\left(\operatorname{Ln} P_{K}\right)+0,0418\left(\operatorname{Ln} P_{G}\right)$

Esto indica que para el rango de la muestra la industria opera con rendimientos a escala decrecientes, considerando que el grado de homogeneidad de la función de producción (s) define el tipo de rendimientos a escala y se relaciona con la función de costos de la siguiente manera (ecuación 7).

$$
s=C M e / C M g=((C / q)) /((\partial C / \partial q))=1 / \varepsilon_{C, Q}
$$

Se detectan deseconomías de escala o rendimientos a escala decrecientes, puesto que para los valores muestrales $s$ toma un valor mínimo de 0,7274 y un valor máximo de 0,8094 , siendo su media 0,7557 . La interpretación conceptual es que para el rango de output en el que operan las empresas concesionarias, el costo medio incrementa al aumentar la producción de servicios (figura 1). En otras palabras, desde la perspectiva de la producción, ante aumentos en igual proporción de los factores productivos, la producción se incrementa en menor proporción. 
Es importante aclarar que esta estimación hace referencia a la estructura de costos de una empresa concesionaria representativa del mercado de transporte colectivo mediante buses del Área Metropolitana de Mendoza, que presta un único servicio (único output) expresado en kilómetros recorridos por año. Los rendimientos decrecientes a escala están presentes en cada firma y su análisis constituye uno de los instrumentos más relevantes para conocer la relación existente entre los costos y la tecnología de producción. En el mercado de transporte colectivo analizado, la tecnología de producción hace referencia a la infraestructura utilizada para la guarda, mantenimiento y reparación de vehículos (vinculada fuertemente al tamaño del parque móvil), a la capacidad de cada uno de ellos, al tamaño de la flota y a la capacidad de gestión de la firma.

Dado que este es un análisis de largo plazo y que las empresas minimizan sus costos, los rendimientos a escala decrecientes muestran que sería óptimo una infraestructura y/o equipamiento vehicular de menor capacidad, es decir, un tamaño de firma menor. Se está operando en un nivel de servicio como $Q_{1}$ y en realidad lo óptimo sería $Q_{0}$ (figura 1). Es decir, si bien cada firma minimiza sus costos, el nivel de servicios prestados por cada una de ellas es mayor a aquel que le permitiría operar con economías de escala.

Figura 1

Curva de costo promedio a largo plazo

Fuente: elaboración propia con base en Zugarramurdi et al. (1998, sección 5.4).

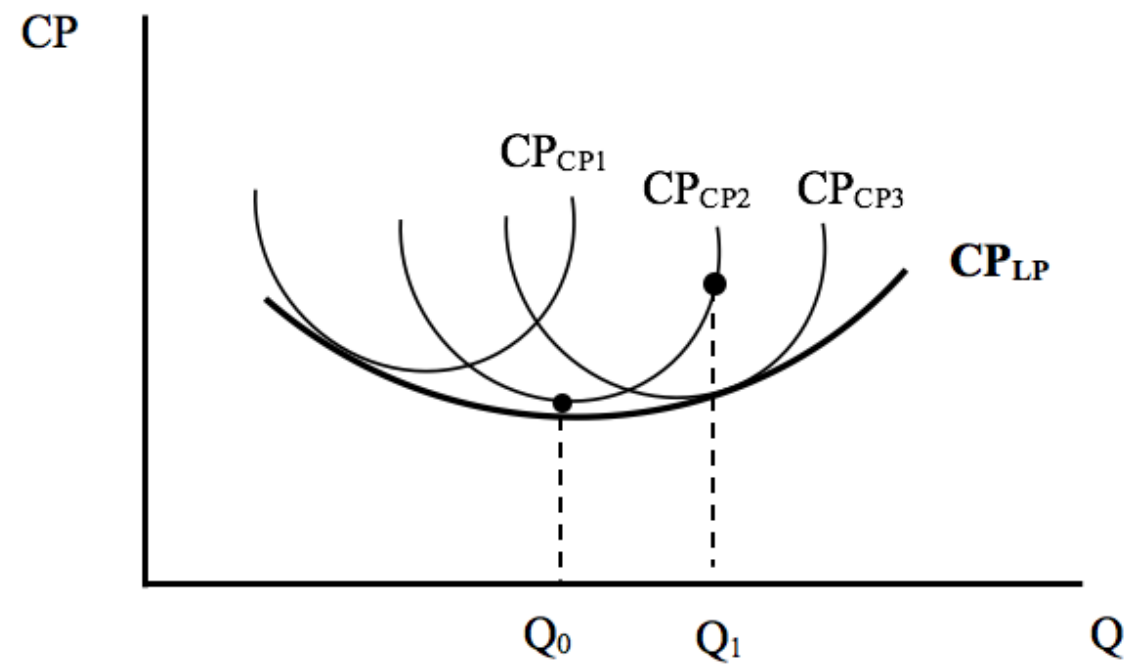


Para conocer el nivel de servicios que cada empresa debería producir por año para tener economías de escala constante se considera la media de todas las variables, excepto la media de producción, y se resuelve la ecuación $\varepsilon_{c, 0}=1$. El resultado indica que los kilómetros anuales que se deberían recorrer son 144.365. Este nivel es mucho menor al que como promedio recorren los operadores actualmente $(7.077 .743 \mathrm{~km})$.

Se asume que el nivel total de servicios que el Estado define como oferta es el óptimo social, aquel que la sociedad demanda para desplazarse fluidamente por el espacio urbano. Bajo este supuesto, el modelo estimado indica que el mercado podría operar con economías de escala si la oferta estuviera más atomizada; es decir, si cada operador ofreciera una cantidad menor de servicios, si cada operador se constituyera como una empresa más pequeña.

\section{CONCLUSIONES}

La existencia de deseconomías de escala puede tener su origen en aspectos internos o externos a la firma. Los primeros son propios de las características particulares de producción de la firma, pueden ser tecnológicos o pecuniarios. Por su parte, los segundos pueden ser monetarios o tecnológicos. Las firmas concesionarias no tienen a su alcance modificar los aspectos externos, mientras que sí pueden decidir sobre los internos.

Los aspectos internos tecnológicos están relacionados con la dimensión de las instalaciones fijas, a la capacidad de los vehículos utilizados para proveer el servicio y al tamaño de la flota. Hacen referencia a una característica propia de la función de producción. Los aspectos internos pecuniarios son relativos a las técnicas de gestión de la empresa, al incremento de costos administrativos debido al inadecuado manejo de la organización o a la mala coordinación de actividades en una escala más grande o al crecimiento de la burocracia.

Los aspectos externos monetarios están asociados a incrementos en los precios de los insumos causados por la ampliación de las empresas que los utilizan e incrementan su precio como efecto de una mayor demanda. Los aspectos externos tecnológicos tienen que ver con el incremento de los costos por razones externas a las firmas; en el caso del transporte colectivo puede deberse al aumento de la congestión vehicular o el mal estado de la infraestructura vial. 
En el caso en estudio, con respecto a las causas internas tecnológicas, se detecta que las firmas tienen un tamaño mayor al óptimo. Esto significa que los costos unitarios se reducirían si la producción media de cada una de ellas fuera menor. Para mejorar esta estructura de costos se requeriría una reducción de la capacidad de la infraestructura para guarda y mantenimiento del parque móvil (o un uso más eficiente del espacio físico disponible) o una reducción de la capacidad de la flota en su conjunto.

En el mercado de transporte colectivo automotor se debe prestar atención particularmente a las características de los vehículos y las condiciones de su explotación, en tanto estas resultan en indivisibilidades en la oferta del servicio. Las firmas prestatarias del servicio no pueden incrementar su oferta de manera continua, sino en bloques definidos por la capacidad de los vehículos (de Rus et al., 2003). Esta característica se puede flexibilizar al contar con vehículos de distintas capacidades, no incurriendo en costos innecesarios. Así, las deseconomías de escala podrían transformarse en economías de escala. Según Iturriza (1982, p. 58) "el tamaño de vehículo en el transporte automotor está asociado con la posibilidad de aprovechar economías originadas en el aumento menos que proporcional de los insumos requeridos para su funcionamiento".

La oferta del servicio de transporte colectivo mediante buses que opera en el Área Metropolitana de Mendoza no se ajusta adecuadamente a la demanda, incurriendo en costos innecesarios debido a dichas indivisibilidades y, por ende, siendo una de las causas de las deseconomías de escala. Los recorridos no se encuentran jerarquizados y los vehículos que circulan por estas rutas tienen todos la misma capacidad, por lo que trayectos de mayor y menor demanda son abastecidos por el mismo tipo de equipamiento, resultando en el segundo caso en capacidad ociosa. Los recorridos troncales, que satisfacen la demanda de corredores de mayor tráfico, requieren de unidades móviles de mayor capacidad, mientras que los recorridos alimentadores, que abastecen áreas menos densas, requieren de unidades de menor tamaño. Esta jerarquización sería un avance hacia la operación del mercado en el tramo de rendimientos a escala constantes, ya que mejora la estructura general de costos y el costo promedio por kilómetro recorrido disminuiría.

Con respecto a las causas internas pecuniarias, en el caso en estudio se observa, por un lado, que las firmas no tienen incentivos económicos para optimizar su funcionamiento, ya que el riesgo es asumido completamente por el Estado, que a su vez cubre la diferencia entre beneficios y costos sin 
condicionamientos. Además, se evidencia que los procedimientos administrativos para cumplir con los requerimientos regulatorios establecidos por la autoridad de aplicación son poco claros y excesivamente burocráticos. Este último factor está muy relacionado a la gestión estatal, por lo que excede las posibilidades de corrección por parte de las empresas operadoras. Así mismo, como ya se mencionó, en el servicio prestado en el Área Metropolitana de Mendoza, la capacidad máxima del vehículo presenta un límite más allá del cual solo puede repetirse un esquema productivo similar, mediante la incorporación de nuevas unidades. Por ello, las características de producción están vinculadas a la capacidad máxima de los vehículos empleados. En esta situación, las economías de escala que logre aprovechar la firma están fuertemente determinadas por la administración empresaria. Bajo esta consideración, cualquier firma pequeña, organizada eficientemente, puede competir con una gran empresa si cuenta con equipos de igual nivel tecnológico. Obeng (1985), en su estudio sobre costos de transporte, afirma que en todos los sistemas de transporte los costos pueden reducirse mejorando la productividad de los factores, inclusive la administración de la empresa, enfocándose en el control de costos en las horas pico.

En referencia al modo de distribución de la recaudación, se observa que este tampoco genera incentivos para la eficiencia en la prestación del servicio. El ingreso principal de cada concesionaria es el boleto que abona cada pasajero, y dado que la empresa recaudadora distribuye estos beneficios según los kilómetros recorridos por cada operador, el riesgo de la actividad lo asume completamente el Estado; es decir, las firmas concesionarias no tienen incentivos para mejorar su servicio y así captar más usuarios, sino que simplemente se limitan a cumplir con las restricciones impuestas por la autoridad de aplicación, entre ellas la cantidad de kilómetros que deben recorrer. Adicionalmente, los subsidios a la oferta sin condicionamientos no mejoran esta situación, dado que no imponen requerimientos técnicos o económicos para el otorgamiento de los mismos. De esta manera, tampoco funcionan como promotores de la mejora en la rentabilidad y en la gestión de las firmas.

Existe otro aspecto de la regulación estatal que acentúa la operación con deseconomías de escala vinculado a la duración de la concesión y a la cantidad de empresas asignatarias. Se observa que la licitación para adjudicar el servicio se realiza cada 10 años, período durante el cual existe esta fuerte barrera de entrada. La cantidad de empresas que operan en el 
mercado viene determinada en parte por el Estado, al definir la cantidad de grupos de recorridos que licitará (a cada grupo se le asigna un operador) y, en parte, por las empresas que están en condiciones de postularse en la licitación en más de un grupo de recorridos. En la licitación vigente durante 1991-2005 las empresas operadoras eran en total 11, las cuales prestaban el servicio de 15 grupos de recorridos. En la licitación siguiente, la concesión se encuentra mucho más concentrada, siendo las firmas adjudicatarias en total 7, prestando el servicio de 10 grupos de recorridos; es decir, se redujo tanto la cantidad de empresas prestatarias como la cantidad de grupos de recorridos que constituyen el servicio de transporte colectivo mediante ómnibus. Ante este fenómeno, el Estado puede generar medidas para mitigar las deseconomías de escala. Por un lado, puede hacer más eficiente el proceso licitatorio para cumplir con el período de concesión; en ambas licitaciones se excedieron los 10 años pautados. Puede, también, acortar este período, de tal manera que sea posible levantar las barreras de entrada a la industria y someter con mayor frecuencia el proceso de competencia por licitación. Por otro lado, puede impedir que una misma empresa se postule para prestar el servicio en más de un grupo de recorridos y puede ampliar la cantidad de grupos recorridos a licitar. Con lo anterior se generan incentivos para atomizar la oferta del servicio.

En cuanto a las causas externas que generan deseconomías de escala, no se detectan aquellas relacionadas a los fenómenos monetarios. Ninguna de las empresas concesionarias posee en los mercados de insumos una participación tal que sus incrementos en compras provoquen traslados de la demanda. En cambio, se observan algunas causas externas tecnológicas que provocan las economías de escala con rendimientos decrecientes. Por un lado, la adquisición de vehículos está condicionada a la política cambiaria y a la restricción a las importaciones existentes en el territorio argentino. Dicho equipamiento se compra en concesionarias nacionales que adquieren los vehículos en el exterior. En el período analizado, las limitaciones a las compras en el exterior han impactado el costo de las nuevas unidades, incrementando consecuentemente los costos totales de las firmas. Por otro lado, se incrementa la estructura de costos de las concesionarias por la congestión vehicular en las horas pico, que resulta cada vez más perjudicial para la circulación de las unidades de transporte colectivo, o por el inadecuado diseño de la red de transporte respecto de los requerimientos de movilidad de la población urbana o de la estructura del sistema urbano. 
Como se ha mencionado, es importante conocer la estructura de mercado y el funcionamiento del mismo para poder establecer instrumentos o mecanismos que incentiven la optimización del uso de los recursos de las empresas concesionarias. Esto se obtiene no solamente a través de estudios similares al presente, sino también a partir de análisis interdisciplinarios con información completa, de calidad y actualizada. Esto, en el caso en estudio, se puede dar en el marco de las leyes sobre ordenamiento territorial sancionadas en los años 2009 y 2017, que darían el sustento legal y administrativo para una integración y articulación entre el servicio de transporte colectivo y el territorio en el que este opera.

Por último, vale la comparación con otro caso latinoamericano, el Sistema Transantiago, Chile (de Grange et al., 2018). En este se detecta que operadores de mayor tamaño tienen mayores costos operativos promedio que prestatarios de menor tamaño. Es decir, resulta deseable una mayor atomización del servicio. La gran diferencia con el servicio prestado en el Área Metropolitana de Mendoza es que los operadores de Transantiago resultan todos empresas de gran envergadura (la firma de menor tamaño posee más de 400 vehículos), mientras que en el territorio en estudio ninguna empresa llega a tener tal capacidad en parque móvil. Lo anterior significa que el Sistema Transantiago puede justificar las deseconomías de escala por el tamaño de los concesionarios, los cuales, según definen Matas y Raymond (1998) y Obeng (1985), son más probables cuando las firmas son grandes. En cambio, en el Área Metropolitana de Mendoza las deseconomías de escala pueden aparecer por otras causas, aunque, según estos autores, no exclusivamente por el tamaño de los oferentes.

Todas estas consideraciones resultan de gran valor para elaborar y diseñar la nueva licitación, favoreciendo así la operación con mayor eficiencia de todos los modos de transporte y del sistema de movilidad en su conjunto. Son aspectos característicos significativos del mercado de transporte colectivo mediante buses del área metropolitana estudiada que, de ser tenidos en cuenta, potenciarían los impactos positivos de la política pública de movilidad futura.

\section{REFERENCIAS}

Asociación Unida Transporte Automotor Mendoza [AUTAM]. (2009). Costos y Tarifas del Transporte Urbano de Pasajeros (2009). AUTAM. 
Berechman, J., \& Giuliano, G. (1985). Economies of scale in bus transit: A review of concepts and evidence. Transportation, 12(4), 313-332. https://doi.org/10.1007/BF00165470

Christensen, L., \& Greene, W. (1976). Economies of scale in U.S. electric power generation. Journal of Political Economy, 84(4), 655-676. https://doi. org/10.1086/260470

Cubukcu, K. M. (2008). Examining the cost structure of urban bus transit industry: Does urban geography help? Journal of Transport Geography, 16(4), 278-291. https://doi.org/10.1016/j.jtrangeo.2007.09.001

De Borger, B. (1984). Cost and productivity in regional bus transportation: The Belgian case. The Journal of Industrial Economics, 37(1), 35-54. https://doi.org/10.2307/2098423

De Grange, L., Troncoso, R., \& Briones, I. (2018). Cost, production and efficiency in local bus industry: An empirical analysis for the bus system of Santiago. Transportation Research Part A: Policy and Practice, 108, 1-11. https://doi.org/10.1016/j.tra.2017.12.007

De Rus, G., Campos, J., \& Nombela, G. (2003). Economía del transporte. Antoni Bosch.

De Rus, G. (2006). The prospects for competition. En J. A. Gómez-lbáñez \& G. de Rus (eds.), Competition in the railway industry: An international comparative analysis (pp. 177-191). Edward Elgar Publishing.

Filippini, M., \& Prioni, P. (2003). The influence of ownership on the cost of bus service provision in Switzerland. An empirical illustration. Applied Economics, 35, 683-690. https://doi.org/10.1080/0003684032000056788

Hensher, D. (1987). Productive efficiency and ownership of urban bus services. Transportation, 14, 209-225. https://doi.org/10.1007/ BF00837530

Iturriza, J. (1982). Las economías de escala en el transporte. Integración Latinoamericana, 68, 54-62.

Jara-Díaz, S. (1982). The estimation of transport cost functions: A methodological review. Transport Reviews, 2(3), 257-278. https://doi. org/10.1080/01441648208716498

Koshal, R. (1970). Economies of scale in bus transport: Il: Some Indian experience. The Journal of Transport Economics and Policy, 4(1), 15-28. 
Lai, L. W., Chau, K. W., \& Cheung, P. A. C. (2012). Scale economies of the franchised buses and ferries of Hong Kong, 1948-1998. Rev. Urban Reg. Develop. Stud., 24(3), 121-140. https://doi.org/10.1111/rurd.12002

Lee, N., \& Steedman, I. (1970). Economies of scale in bus transport: I. Some British municipal results. Journal of Transport Economics and Policy, $4(1), 29-36$.

Matas, A., \& Raymond, J. L. (1998). Technical characteristics and efficiency of urban bus companies: The case of Spain. Transportation, 25, 243-263. https://doi.org/10.1023/A:1005078830008

Miller, D. (1970). Differences among cities, differences among firms, and costs of urban bus transport. Journal of Industrial Economics, 19(1), 2232. https://doi.org/10.2307/2097534

Obeng, K. (1985). Bus transit cost, productivity and factor substitution. Journal of Transport Economics and Policy, 19(2), 183-203.

Shepard, R. W. (1970). Theory of cost and production function. Princeton University Press.

Tauchen, H., Fravel, D. F., \& Gilbert, G. (1983). Cost structure of the intercity bus industry. The Journal of Transport Economics and Policy, 17(1), 25-47.

Troncoso, R. (2017). Deseconomías de escala en el transporte de buses de Santiago, Chile. Análisis Centro de Políticas Públicas, n. ${ }^{\circ}$ 1, Universidad del Desarrollo, Santiago de Chile.

Viton, P. (1981). A translog cost function for urban bus transit. J. Indust. Econ., 29(3), 287-304. https://doi.org/10.2307/2098305

Williams, M., \& Dalal, A. (1981). Estimation of the elasticities of factor substitution in urban bus transportation: A cost function approach. Journal of Regional Science, 21(2), 263-275. https://doi. org/10.1111/j.1467-9787.1981.tb00698.x

Zellner, A. (1962). An efficient method of estimating seemingly unrelated regressions and tests for aggregation bias. Journal of the American Statistical Society, 57, 585-612. https://doi.org/10.1080/01621459.19 62.10480664

Zugarramurdi, A., Parín, M., \& Lupin, H. (1998). Ingeniería económica aplicada a la industria pesquera. Documento técnico n. 351 . Organización de las Naciones Unidas para la Agricultura y la Alimentación. 